\title{
Impact of non-digestible carbohydrates on biomarkers of gastrointestinal health: a human intervention study
}

\author{
N. D. Willis ${ }^{1}$, S. Mann ${ }^{2}$, L. Xie ${ }^{1}$, I. D. J. McCallum ${ }^{1}$, S. B. Kelly ${ }^{3}$, D. M. Bradburn ${ }^{4}$, N. J. Belshaw ${ }^{2}$, \\ I. T. Johnson ${ }^{2}$ and J. C. Mathers \\ ${ }^{1}$ Human Nutrition Research Centre, Institute for Ageing and Health, Newcastle University, Campus for Ageing and Vitality, \\ Newcastle-upon-Tyne, NE4 5PL, UK, ${ }^{2}$ Institute of Food Research, Norwich Research Park, Colney, Norwich, NR4 7UA, \\ UK, ${ }^{3}$ North Tyneside General Hospital, North Shields, Tyne and Wear, NE29 8NH, UK and ${ }^{4}$ Wansbeck General Hospital, \\ Ashington, Northumberland, NE63 9JJ, UK
}

Epidemiological evidence shows that dietary choices influence risk of developing sporadic colorectal cancer (CRC), a disease associated strongly with ageing ${ }^{(1)}$. Identification of beneficial dietary agents for cancer prevention requires intervention studies, but progress has been limited by the lack of reliable biomarkers for use as surrogate endpoints. We have developed a panel of novel biomarkers of diet-related CRC risk ${ }^{(2,3,4)}$. This study aimed to test the hypothesis that these biomarkers are responsive to dietary intervention.

Seventy-five healthy participants took part in a randomised, placebo-controlled trial comparing two non-digestible carbohydrates (NDCs), resistant starch and polydextrose. Participants were randomised to the NDCs and appropriate placebos using a $2 \times 2$ factorial design with 4 treatments identified only as A, B, C and D. Participants were stratified according to endoscopy procedure and took the treatment for 50 days. Rectal mucosal biopsies, blood, urine and stool were collected pre- and post-intervention to assess biomarkers related to nutritional and inflammatory status, NDC fermentation, methylation of cancer-related genes, crypt cell kinetics and expression of cell cycle genes. Participant demographics, anthropometrics and habitual diet were also recorded. At present, the study remains blinded.

At baseline the mean age of participants was significantly higher in treatment group B, compared with all other treatment groups $(P=0.043)$. No significant difference in gender distribution, participant smoking status or anthropometrics, including BMI, was detected. The treatment group had no effect on circulating vitamin B12 $(P=0.652)$, red cell folate $(P=0.877)$ or selenium $(P=0.989)$ status, however participants were notably well-supplied with selenium at baseline. Participant weight, BMI, hip, waist and thigh circumference did not change over the course of the intervention.

Recruitment to this study is now complete and we report that phenotypic markers, including body measurements and nutritional status, were not affected by treatment. NDCs are thought to exert anti-neoplastic effects in the colon via the production of butyrate as a result of bacterial fermentation, thereby reducing inflammation. Systemic inflammation levels (as measured by hsCRP) did not appear to change in response to treatment group. Faecal concentrations of calprotectin are currently being assessed to determine the impact of NDC on inflammation localised to the large bowel.

1. WCRF/AICR (2007) Food, Nutrition, Physical Activity and the Prevention of Cancer: a global perspective.

2. Polley ACJ, Mulholland F, Pin C et al. (2006) Cancer Res 66, 6553-6562.

3. Belshaw NJ, Elliott GO, Foxall RJ et al. (2008) Br J Cancer 99, 136-142.

4. Dronamraju SS, Coxhead JM, Kelly SB et al. (2009) Gut 58, 413-420. 